Research Article

\title{
Simultaneity of Synchronization and Antisynchronization in a Class of Chaotic Systems
}

\author{
Zhi Liu, ${ }^{1}$ Rongwei Guo $\mathbb{D}^{2},{ }^{2}$ Yi Qi $\mathbb{D}^{3},{ }^{3}$ and Cuimei Jiang $\mathbb{D}^{2}$ \\ ${ }^{1}$ School of Information Engineering, Key Laboratory of TCM Data Cloud Service in Universities of Shandong, \\ Shandong Management University, Jinan 250357, China \\ ${ }^{2}$ School of Mathematics and Statistics, Qilu University of Technology (Shandong Academy of Sciences), Jinan 250353, China \\ ${ }^{3}$ Texas South University, Houston 250061, USA \\ Correspondence should be addressed to Rongwei Guo; rongwei_guo@163.com
}

Received 11 April 2020; Revised 26 June 2020; Accepted 2 July 2020; Published 22 July 2020

Academic Editor: Alessio Gizzi

Copyright (c) 2020 Zhi Liu et al. This is an open access article distributed under the Creative Commons Attribution License, which permits unrestricted use, distribution, and reproduction in any medium, provided the original work is properly cited.

\begin{abstract}
In this paper, a new synchronization phenomenon, that is, the simultaneity of synchronization and antisynchronization, is investigated for a class of chaotic systems. First, for a given chaotic system, necessary and sufficient conditions for the simultaneity of synchronization and antisynchronization are proved. Then, based on these conditions, all solutions of such synchronization phenomenon for a given chaotic system are derived. After that, physical controllers that are not only simple but also implementable are designed to realize the simultaneity of synchronization and antisynchronization in the above system. Finally, illustrative examples based on numerical simulations are used to verify the validity and effectiveness of the above theoretical results.
\end{abstract}

\section{Introduction}

Lorenz firstly proposed the famous Lorenz chaotic system in 1963. From then on, many researchers are stimulated to investigate the chaotic phenomena, and lots of chaotic systems and hyperchaotic systems are obtained. Since Pecora and Carroll first proposed the chaos synchronization method [1], meanwhile Ott et al. first obtained the chaos control method [2] in 1990, chaotic systems and their control problems have attracted significant attention and have been investigated extensively [3-17]. However, most of the existing works focus on investigating the same kind of synchronization in a given system, i.e., all the states of the master system have the same kind of synchronization with the corresponding states of the slave system. For example, when two systems are referred to as completely synchronized with each other, it means that each pair of the states between the interactive systems is completely synchronous.

In general, coexistence problem is very common, see [18-22] and the references therein. Recently, a new synchronization phenomenon, that is, the simultaneity of synchronization and antisynchronization, was firstly found in the generalized Lorenz system [23]. In this system, the variables $x_{1}, x_{2}$ of the master system antisynchronize the variables $y_{1}, y_{2}$ of the slave system, while the variable $x_{3}$ of the master system synchronizes the variable $y_{3}$ of the slave system. This new synchronization phenomenon is very interesting not only in applications but also in theory. The existence of the simultaneity of synchronization and antisynchronization problem is a basis for designing a physical controller. Zhang et al. [23] found this new synchronization phenomenon, but they did not address some critical theoretical questions. For instance, for a given chaotic system, how to prove the existence of the simultaneity of synchronization and antisynchronization? In addition, if such new synchronization phenomenon exists, how many solutions can be derived. Therefore, for a given chaotic system, it is critical to have a systematic method that can be used for proving the simultaneity of synchronization and antisynchronization, deriving all solutions for this type of synchronization phenomenon and then designing the corresponding physical controller. To address this critical problem, this study is to develop such a systematic method that can be applied to a class of chaotic systems. 
To realize the simultaneity of synchronization and antisynchronization in a chaotic system, many methods can be used, including the linear feedback control method, nonlinear feedback control method, and dynamic feedback control method. Among those methods, the dynamic feedback control method has been widely applied and was adopted by this study.

In this paper, we investigated the simultaneity of synchronization and antisynchronization problem in a class of chaotic systems by using the dynamic feedback control method. At first, the existence of the simultaneity of synchronization and antisynchronization problem of such system is proved. Then, physical controllers were designed to realize the simultaneity of synchronization and antisynchronization in such system. After that, numerical simulations were conducted to verify the validity and correctness of the obtained theoretical results.

\section{Preliminary}

Consider the following chaotic system:

$$
\dot{X}=F(X),
$$

where $X \in R^{n}$ is the state, $F(X) \in R^{n}$ is a continuous vector function, i.e.,

$$
\begin{aligned}
X & =\left(\begin{array}{c}
X^{E} \\
X^{e}
\end{array}\right), \\
F(X) & =\left(\begin{array}{l}
F^{E}(X) \\
F^{e}(X)
\end{array}\right)=\left(\begin{array}{c}
F^{E}\left(X^{E}, X^{e}\right) \\
F^{e}\left(X^{E}, X^{e}\right)
\end{array}\right),
\end{aligned}
$$

$X^{E} \in R^{m}, X^{e} \in R^{n-m}, \quad m \geq 1, \quad F^{E}(X) \in R^{m}$, and $F^{e}(X) \in$ $R^{n-m}$.

For system (1), the slave system can be described as

$$
\dot{Y}=F(Y)+B u,
$$

where $Y \in R^{n}$ is the state, $F(Y) \in R^{n}$ is a continuous vector function, $B \in R^{n \times r}$ is a constant matrix, $r \geq 1$, and $u \in R^{r}$ is the controller to be designed, i.e.,

$$
\begin{aligned}
Y & =\left(\begin{array}{c}
Y^{E} \\
Y^{e}
\end{array}\right), \\
B & =\left(\begin{array}{c}
B^{E} \\
B^{e}
\end{array}\right), \\
F(Y) & =\left(\begin{array}{l}
F^{E}(Y) \\
F^{e}(Y)
\end{array}\right)=\left(\begin{array}{c}
F^{E}\left(Y^{E}, Y^{e}\right) \\
F^{e}\left(Y^{E}, Y^{e}\right)
\end{array}\right),
\end{aligned}
$$

where

$$
Y^{E} \in R^{m}, Y^{e} \in R^{n-m},
$$

$m \geq 1$,

$F^{E}(Y) \in R^{m}$, and $F^{e}(Y) \in R^{n-m}$.

Let $E^{E}=X^{E}+Y^{E}$ and $E^{e}=Y^{e}-X^{e}$, then the sum and error system can be described as follows:

$$
\dot{E}=G(X, Y, E)+B u,
$$

where $E \in R^{n}$ is the state:

$$
\begin{aligned}
E & =\left(\begin{array}{c}
E^{E} \\
E^{e}
\end{array}\right), \\
G(X, Y, E) & =\left(\begin{array}{c}
G^{E}(X, Y, E) \\
G^{e}(X, Y, E)
\end{array}\right) \\
& =\left(\begin{array}{c}
F^{E}\left(Y^{E}, Y^{e}\right)+F^{E}\left(X^{E}, X^{e}\right) \\
F^{e}\left(Y^{E}, Y^{e}\right)-F^{e}\left(X^{E}, X^{e}\right)
\end{array}\right), \\
\dot{E}^{E} & =F^{E}\left(Y^{E}, Y^{e}\right)+F^{E}\left(X^{E}, X^{e}\right)+B^{E} u, \\
\dot{E}^{e} & =F^{e}\left(Y^{E}, Y^{e}\right)-F^{e}\left(X^{E}, X^{e}\right)+B^{e} u,
\end{aligned}
$$

where $B$ is given by equation (4).

Next, a definition is presented as follows.

Definition 1. Consider the sum system (9) and the error system (10). If $\lim _{t \rightarrow \infty}\left\|E^{E}(t)\right\|=0$ and $\lim _{t \rightarrow \infty}\left\|E^{e}(t)\right\|=0$, then the master system (1) and the slave system (3) are called to achieve the simultaneity of synchronization and antisynchronization.

To present the proposed method, a lemma is introduced at first.

Lemma 1 (see [17]). Consider the following system:

$$
\dot{x}=h(x)+b u,
$$

where $x \in R^{n}$ is the state, $h(x) \in R^{n}$ is a vector function, $b \in R^{n \times l}$ is a constant matrix, $l \geq 1$, and $u \in R^{l}$ is the controller to be designed. If $(h(x), b)$ can be stabilized, then a dynamic feedback controller $u$ is designed as follows:

$$
u=K x \text {, }
$$

where $K=k(t) b^{T}$, and the feedback gain $k(t)$ is updated by the following law:

$$
\dot{k}(t)=-\|x(t)\|^{2} .
$$

\section{Problem Formulation}

Consider the following chaotic system:

$$
\dot{x}=f(x) \text {, }
$$

where $x \in R^{n}$ is the state and $f(x) \in R^{n}$ is a vector function.

The main goal of this paper is to investigate the simultaneity of synchronization and antisynchronization for the given chaotic system (14) in the following three aspects:

(1) The existence of the simultaneity of synchronization and antisynchronization.

(2) The solutions of the simultaneity of synchronization and antisynchronization for this chaotic system.

(3) The implementation of the simultaneity of synchronization and antisynchronization. A simple and physically implementable controller is designed for such a problem. 


\section{Main Result}

4.1. Existence of the Simultaneity of Synchronization and Antisynchronization. In this section, the necessary and sufficient conditions for the existence of the simultaneity of synchronization and antisynchronization are proved for two given chaotic systems.

Theorem 1. Consider the chaotic system (1). Its existence of the simultaneity of synchronization and antisynchronization can be realized by the controllers in the following form:

$$
\begin{aligned}
u & =H\left(E^{E}, E^{e}, X\right), \\
H(0,0, X) & =0,
\end{aligned}
$$

if and only if

$$
\begin{aligned}
& F^{E}\left(X^{E}, X^{e}\right)=-F^{E}\left(-X^{E}, X^{e}\right), \\
& F^{e}\left(X^{E}, X^{e}\right)=F^{E}\left(-X^{E}, X^{e}\right) .
\end{aligned}
$$

Proof (necessity). According to the nonlinear control theorem, $E^{E}=0$ and $E^{e}=0$ should be the equilibria of the following systems:

$$
\begin{aligned}
\dot{E}^{E} & =F^{E}\left(Y^{E}, Y^{e}\right)+F^{E}\left(X^{E}, X^{e}\right) \\
& =F^{E}\left(E^{E}-X^{E}, E^{e}+X^{e}\right)+F^{E}\left(X^{E}, X^{e}\right), \\
\dot{E}^{e} & =F^{e}\left(Y^{E}, Y^{e}\right)-F^{e}\left(X^{E}, X^{e}\right) \\
& =F^{e}\left(E^{E}-X^{E}, E^{e}+X^{e}\right)-F^{E}\left(X^{E}, X^{e}\right),
\end{aligned}
$$

respectively.

It results in

$$
\begin{aligned}
& F^{E}\left(-X^{E}, X^{e}\right)+F^{E}\left(X^{E}, X^{e}\right)=0, \\
& F^{e}\left(-X^{E}, X^{e}\right)-F^{E}\left(X^{E}, X^{e}\right)=0,
\end{aligned}
$$

i.e., equations (16) and (17) hold.

Proof (sufficiency). If equations (16) and (17) hold, which implies that $E^{E}=0$ and $E^{e}=0$ are the equilibria of systems (18) and (19), respectively. Thus, the simultaneity of synchronization and antisynchronization for the given chaotic system can be realized by the controller $u$ given in equation (15).

Especially, if

$$
F(X)=\left(\begin{array}{c}
F^{E}\left(X^{E}, X^{e}\right) \\
F^{e}\left(X^{E}, X^{e}\right)
\end{array}\right)=\left(\begin{array}{c}
M\left(X^{e}\right) X^{E} \\
N\left(X^{E}\right) X^{e}
\end{array}\right),
$$

where $N\left(-X^{E}\right)=N\left(X^{E}\right)$, system (1) becomes

$$
\begin{aligned}
& \dot{X}^{E}=M\left(X^{e}\right) X^{E}, \\
& \dot{X}^{e}=N\left(X^{E}\right) X^{e},
\end{aligned}
$$

and the simultaneity of synchronization and antisynchronization of system (22) holds.
Theorem 2. Consider the chaotic system (14). Its existence of the simultaneity of synchronization and antisynchronization if and only the following algebraic equation about $\alpha$

$$
\left\{\begin{array}{lll}
f_{1}(\alpha x) & \equiv \alpha_{1} f_{1}(x), \\
f_{2}(\alpha x) & \equiv \alpha_{2} f_{2}(x), \\
\vdots & \equiv \\
f_{n}(\alpha x) & \equiv \alpha_{n} f_{n}(x),
\end{array}\right.
$$

has a solution in the following form:

$$
\beta^{(s)}=\left(\begin{array}{c}
\alpha_{i_{1}} \\
\vdots \\
\alpha_{i_{s-1}} \\
\alpha_{i_{s}} \\
\alpha_{i_{s+1}} \\
\vdots \\
\alpha_{i_{n}}
\end{array}\right)=\left(\begin{array}{c}
-1 \\
-1 \\
\vdots \\
-1 \\
1 \\
\vdots \\
1
\end{array}\right) \leftarrow s,
$$

where $s \geq 1$ is the number of $\alpha_{i_{j}}=-1$, $i_{j} \in \Lambda=\{1,2, \ldots, n\}, j=1,2, \ldots, n$, and $\alpha$ is given as follows:

$$
\begin{gathered}
\alpha=\left(\begin{array}{ccccc}
\alpha_{1} & 0 & 0 & \cdots & 0 \\
0 & \alpha_{2} & 0 & \cdots & 0 \\
0 & 0 & \alpha_{3} & \cdots & 0 \\
\vdots & \vdots & \vdots & \vdots & \vdots \\
0 & 0 & 0 & \cdots & \alpha_{n}
\end{array}\right), \\
\left|\alpha_{i}\right|=1, \quad i \in \Lambda .
\end{gathered}
$$

Proof. For system (14), the uncontrolled slave system is given as

$$
\dot{y}=f(y),
$$

where $y \in R^{n}$ is the state.

Let $e=y-\alpha x$, where $\alpha$ is given in (25), then the sum and error system is

$$
\dot{e}=f(y)-\alpha f(x)
$$

where $e \in R^{n}$ is the state.

Then, the existence of the simultaneity of synchronization and antisynchronization in system (14) if and only if $e=0$ is the equilibrium point of system (28), i.e.,

$$
f(y)-\alpha f(x)=f(\alpha x)-\alpha f(x) \equiv 0,
$$

which completes the proof.

4.2. Solutions of the Simultaneity of Synchronization and Antisynchronization. We can obtain all solutions of the simultaneity of synchronization and antisynchronization in the given chaotic system (14) by solving equation (23).

After proving the existence of the simultaneity of synchronization and antisynchronization in a given chaotic 
system (1), the question about how to find a nonsingular transformation matrix $T$ to transfer system (14) into system (1) will be raised naturally.

In general, according to the solutions of the algebraic equation (23), we can find the matrix $T$ by the following algorithm:

Algorithm 1. $k=1$; let $s$ be the number of $\alpha_{j}=-1, j \in \Lambda$,

$$
\min \left\{j \mid \alpha_{j}=-1, j \in \Lambda\right\} \triangleq i_{k}
$$

while $k \leq s$ do

$$
\begin{gathered}
k=k+1, \\
\min _{j \in \Lambda}\left\{\alpha_{j}=-1, j \neq i_{1}, i_{2}, \ldots, i_{k-1}\right\} \triangleq i_{k} .
\end{gathered}
$$

Then, let

$$
X^{E}=\left(\begin{array}{c}
X_{1}^{E} \\
X_{2}^{E} \\
\vdots \\
X_{s}^{E}
\end{array}\right)=\left(\begin{array}{c}
x_{i_{1}} \\
x_{i_{2}} \\
\vdots \\
x_{i_{s}}
\end{array}\right) \text {. }
$$

Next,

$$
\begin{aligned}
& k=s+1, \\
& \min \left\{j \mid \alpha_{j}=1, j \in \Lambda\right\} \triangleq i_{k} .
\end{aligned}
$$

while $k \leq n$ do

$$
\begin{aligned}
& k=k+1, \\
& \min \left\{\alpha_{j}=1, j \neq i_{s+1}, i_{s+2}, \ldots, i_{k-1}, j \in \Lambda\right\} \triangleq i_{k} .
\end{aligned}
$$

Then, let

$$
X^{e}=\left(\begin{array}{c}
X_{s+1}^{e} \\
X_{s+2}^{e} \\
\vdots \\
X_{n}^{e}
\end{array}\right)=\left(\begin{array}{c}
x_{i_{s+1}} \\
x_{i_{s+2}} \\
\vdots \\
x_{i_{n}}
\end{array}\right) \text {. }
$$

By Algorithm 1, the nonsingular transform matrix $T$ can be obtained as follows:

$$
\begin{aligned}
& T=\left(\begin{array}{c}
\delta_{n}^{i_{1}} \\
\vdots \\
\delta_{n}^{i_{s}} \\
\vdots \\
\delta_{n}^{i_{n}}
\end{array}\right), \\
& \delta_{n}^{i_{1}}=\left(\begin{array}{ccccccc}
0 & \cdots & 0 & 1 & 0 & \cdots & 0 \\
& & & \uparrow & & & \\
& & & & & & \\
& & & i_{1} & & &
\end{array}\right) \in R^{n},
\end{aligned}
$$

where $i_{j} \in \Lambda, j=1,2, \ldots, n$.
For example, for the chaotic system, $\dot{x}=f(x), x \in R^{3}$, and $f(x) \in R^{3}$. If $\alpha_{1}=-1, \alpha_{2}=1$, and $\alpha_{3}=-1$, then $s=2$, $i_{1}=1, i_{2}=3$, and $i_{3}=2$. By Algorithm 1 , we obtain

$$
T=\left(\begin{array}{c}
\delta_{3}^{i_{1}} \\
\delta_{3}^{i_{2}} \\
\delta_{3}^{i_{3}}
\end{array}\right)=\left(\begin{array}{l}
\delta_{3}^{1} \\
\delta_{3}^{3} \\
\delta_{3}^{2}
\end{array}\right)=\left(\begin{array}{lll}
1 & 0 & 0 \\
0 & 0 & 1 \\
0 & 1 & 0
\end{array}\right) \text {. }
$$

By $T$, the system $\dot{x}=f(x)$ is transferred into the following system:

$$
\dot{X}=F(X) \text {, }
$$

$$
\begin{aligned}
X & =\left(\begin{array}{c}
X_{1} \\
X_{2} \\
X_{3}
\end{array}\right)=\left(\begin{array}{c}
X^{E} \\
X^{e}
\end{array}\right)=T x=\left(\begin{array}{c}
x_{1} \\
x_{3} \\
x_{2}
\end{array}\right), \\
F(X) & =\left(\begin{array}{l}
F_{1}(X) \\
F_{2}(X) \\
F_{3}(X)
\end{array}\right)=\left(\begin{array}{c}
F^{E}\left(X^{E}, X^{e}\right) \\
F^{e}\left(X^{E}, X^{e}\right)
\end{array}\right) \\
& =T f(x)=\left(\begin{array}{c}
f_{1}(x) \\
f_{3}(x) \\
f_{2}(x)
\end{array}\right) .
\end{aligned}
$$

4.3. Implementation of the Simultaneity of Synchronization and Antisynchronization. In this section, a simple and physically implementable controller is designed for the implementation of the simultaneity of synchronization and antisynchronization.

According to the results in [16], we propose the following theorem.

Theorem 3. Consider the sum system (9) and error system (10).

If $(G(X, Y, E), B)$ can be stabilized, then the controller $u$ is designed as follows:

$$
u=K E \text {, }
$$

where $K=k(t) B^{T}$, and $k(t)$ is updated by the following update law:

$$
\dot{k}=-\gamma\|E\|^{2}
$$

and $\gamma>0$, which implies that the master system (1) and the slave system (3) reach the simultaneity of synchronization and antisynchronization.

Proof. Since $(G(X, Y, E), B)$ can be stabilized, according to Lemma 1 , the controller $u$ is designed as equation (44).

\section{Illustrative Examples Based on Numerical Simulations}

In this section, two examples based on numerical simulations are used to demonstrate the validity and effectiveness of the derived theoretical results. 
Example 1. The Lorenz system [24]:

$$
\dot{x}=f(x)=\left(\begin{array}{c}
f_{1}(x) \\
f_{2}(x) \\
f_{3}(x)
\end{array}\right)=\left(\begin{array}{c}
10\left(x_{2}-x_{1}\right) \\
28 x_{1}-x_{2}-x_{1} x_{3} \\
8 \\
-\frac{8}{3} x_{3}+x_{1} x_{2}
\end{array}\right) .
$$

According to the algebraic equation (24),

$$
\left\{\begin{array}{l}
f_{1}(\alpha x)-\alpha_{1} f_{1}(\alpha x)=10\left(\alpha_{2}-\alpha_{1}\right) x_{2} \equiv 0, \\
f_{2}(\alpha x)-\alpha_{2} f_{2}(\alpha x)=28\left(\alpha_{1}-\alpha_{2}\right) x_{1}-\left(\alpha_{1} \alpha_{3}-\alpha_{2}\right) x_{1} x_{3} \equiv 0, \\
f_{3}(\alpha x)-\alpha_{3} f_{3}(\alpha x)=\left(\alpha_{1} \alpha_{2}-\alpha_{3}\right) x_{1} x_{2} \equiv 0,
\end{array}\right.
$$

and it results in

$$
\left\{\begin{array}{l}
\alpha_{2}=\alpha_{1} \\
\alpha_{1} \alpha_{3}=\alpha_{2} \\
\alpha_{1} \alpha_{2}=\alpha_{2}
\end{array}\right.
$$

By solving equation (48), a solo solution can be obtained:

$$
\beta^{(2)}=\left(\begin{array}{c}
\alpha_{i_{1}} \\
\alpha_{i_{2}} \\
\alpha_{i_{3}}
\end{array}\right)=\left(\begin{array}{l}
\alpha_{1} \\
\alpha_{2} \\
\alpha_{3}
\end{array}\right)=\left(\begin{array}{c}
-1 \\
-1 \\
1
\end{array}\right) .
$$

By Algorithm 1, it results in

$$
T=\left(\begin{array}{c}
\delta_{3}^{i_{1}} \\
\delta_{3}^{i_{2}} \\
\delta_{3}^{i_{3}}
\end{array}\right)=\left(\begin{array}{l}
\delta_{3}^{1} \\
\delta_{3}^{2} \\
\delta_{3}^{2}
\end{array}\right)=\left(\begin{array}{lll}
1 & 0 & 0 \\
0 & 1 & 0 \\
0 & 0 & 1
\end{array}\right) .
$$

Thus, let $X^{E}=\left(x_{1}, x_{2}\right)^{T}$ and $X^{e}=x_{3}$; the Lorenz system (46) is rewritten as follows:

$$
\begin{aligned}
\dot{X}^{E} & =F^{E}(X), \\
\dot{X}^{e} & =F^{e}(X), \\
F^{E}(X) & =M\left(X^{e}\right) X^{E}, \\
M\left(X^{e}\right) & =\left(\begin{array}{cc}
-10 & 10 \\
28-X^{e} & -1
\end{array}\right), \\
F^{e}(X) & =-\frac{8}{3} X^{e}+X_{1}^{E} X_{2}^{E} .
\end{aligned}
$$

Then, the slave Lorenz system is given as

$$
\begin{aligned}
\dot{Y} & =F(Y)+B u, \\
Y & =\left(\begin{array}{c}
Y^{E} \\
Y^{e}
\end{array}\right), \\
F(Y) & =\left(\begin{array}{c}
F^{E}(Y) \\
F^{e}(Y)
\end{array}\right),
\end{aligned}
$$

$$
\begin{aligned}
F^{E}(Y) & =M\left(Y^{e}\right) Y^{E}, \\
M\left(Y^{e}\right) & =\left(\begin{array}{cc}
-10 & 10 \\
28-Y^{e} & -1
\end{array}\right), \\
F^{e}(Y) & =-\frac{8}{3} Y^{e}+Y_{1}^{E} Y_{2}^{E}, \\
B=\left(\begin{array}{c}
B^{E} \\
B^{e}
\end{array}\right) & =\left(\begin{array}{c}
B^{E} \\
0
\end{array}\right), \\
B^{E} & =\left(\begin{array}{c}
0 \\
1
\end{array}\right) .
\end{aligned}
$$

Let $E^{E}=X^{E}+Y^{E}$ and $E^{e}=Y^{E}-X^{e}$; then, the sum and error system is given as

$$
\begin{aligned}
& \dot{E}=G(X, Y, E)+B u, \\
& E=\left(\begin{array}{c}
E^{E} \\
E^{e}
\end{array}\right),
\end{aligned}
$$

where $B$ is given by equation (60) and $u$ is the controller to be designed.

Considering the following uncontrolled sum and error system,

$$
\begin{aligned}
& \dot{E}_{1}^{E}=-10 E_{1}^{E}+10 E_{2}^{E}, \\
& \dot{E}_{2}^{E}=28 E_{1}^{E}-E_{2}^{E}-E_{1}^{E} E_{3}^{e}-X_{3}^{e} E_{1}^{E}+X_{1}^{E} E_{3}^{e}, \\
& \dot{E}_{3}^{e}=-\frac{8}{3} E_{3}^{e}+E_{1}^{E} E_{2}^{E}-X_{1}^{E} E_{2}^{E}-X_{2}^{E} E_{1}^{E},
\end{aligned}
$$

if $E_{2}^{E}=0$, then the following system

$$
\begin{aligned}
& \dot{E}_{1}^{E}=-10 E_{1}^{E}, \\
& \dot{E}_{3}^{e}=-\frac{8}{3} E_{3}^{e}-X_{2}^{E} E_{1}^{E},
\end{aligned}
$$

is globally asymptotically stable.

Thus, $(G(X, Y, E), B)$ can be stabilized, and according to Theorem 3, the controller is designed as

$$
\begin{aligned}
u & =K E=k(t) B^{T} E=k(t)\left(\begin{array}{lll}
0 & 1 & 0
\end{array}\right) E=k(t) E_{2}^{E}, \\
\dot{E}_{1}^{E} & =-10 E_{1}^{E}+10 E_{2}^{E}, \\
\dot{E}_{2}^{E} & =28 E_{1}^{E}-E_{2}^{E}-E_{1}^{E} E_{3}^{e}-X_{3}^{e} E_{1}^{E}+X_{1}^{E} E_{3}^{e}+k(t) E_{2}^{E}, \\
\dot{E}_{3}^{e} & =-\frac{8}{3} E_{3}^{e}+E_{1}^{E} E_{2}^{E}-X_{1}^{E} E_{2}^{E}-X_{2}^{E} E_{1}^{E},
\end{aligned}
$$

and $\dot{k}(t)=-\|E\|^{2}$.

To demonstrate the validity and correctness of the derived theoretical results, numerical simulation is conducted by setting the following initial conditions: $x_{1}(0)=1.2, x_{2}(0)=-2.3, \quad x_{3}(0)=3.2$, $y_{1}(0)=5.8, y_{2}(0)=-6.2, y_{3}(0)=7.1, \quad$ and $\quad k(0)=-1$. Figure 1 shows that the sum and error system is asymptotically stable; Figure 2 shows that the states of the master 


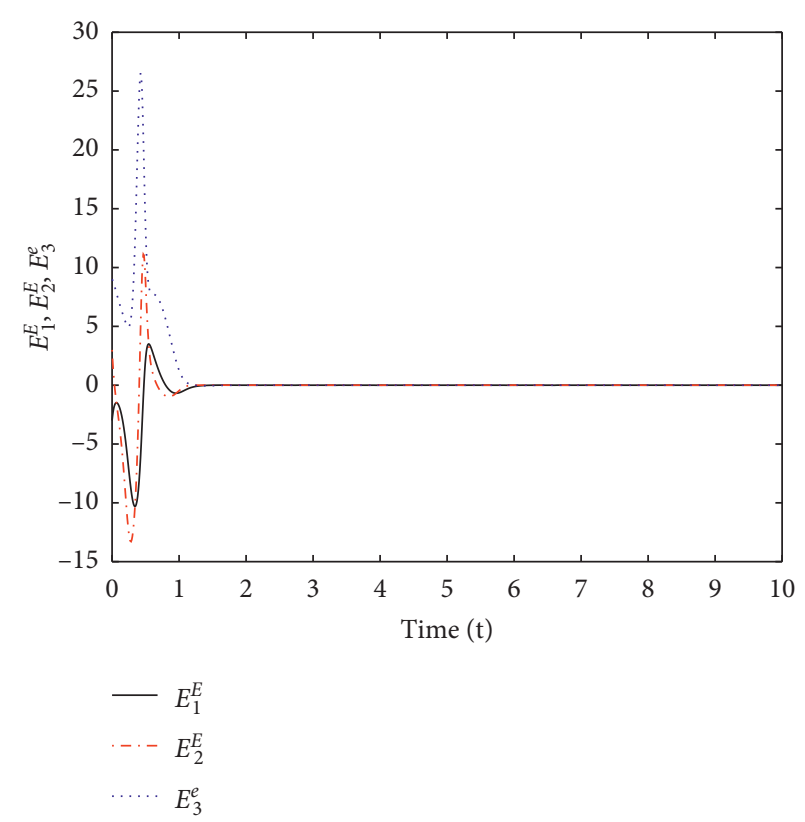

FIgURE 1: The sum and error system is asymptotically stable.
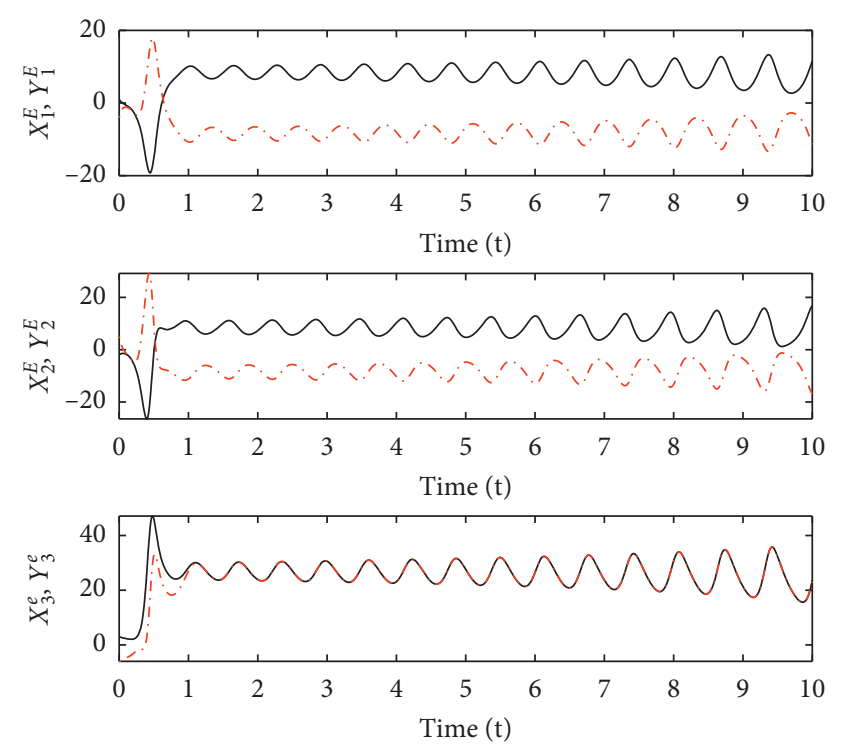

FIgURE 2: $X_{1}^{E}, X_{2}^{E}, X_{3}^{e}$ and $Y_{1}^{E}, Y_{2}^{E}, Y_{3}^{e}$, respectively.

system (49) and the controlled slave system (53), respectively. Figure 3 shows that the feedback gain $k(t)$ tends to a constant.

Example 2. The Chen-Lee system [25]:

$$
\dot{x}=f(x)=\left(\begin{array}{c}
f_{1}(x) \\
f_{2}(x) \\
f_{3}(x)
\end{array}\right)=\left(\begin{array}{c}
-x_{2} x_{3}+5 x_{1} \\
x_{1} x_{3}-10 x_{2} \\
\frac{1}{3} x_{1} x_{2}-3.8 x_{3}
\end{array}\right),
$$

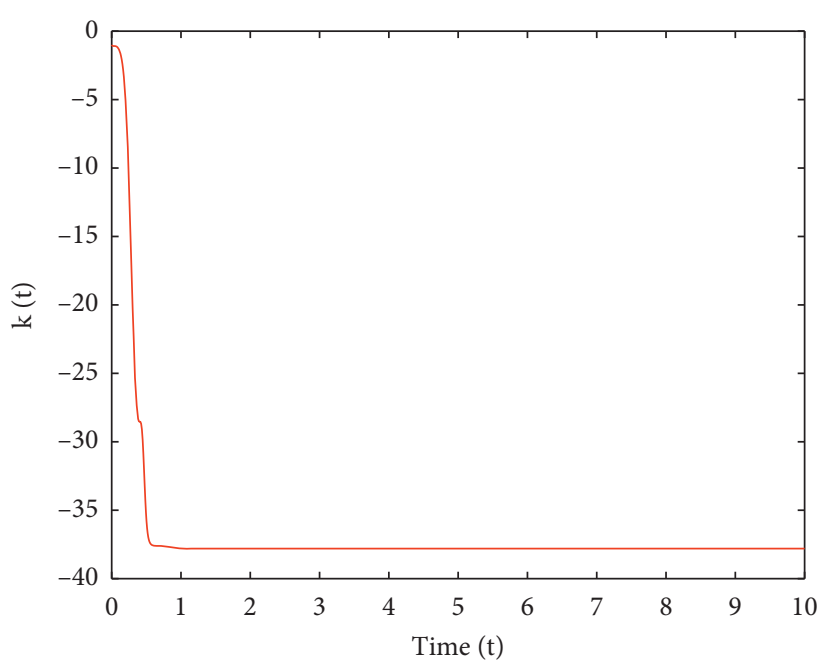

FIgURE 3: $k(t)$ tends to a constant.

where $x \in R^{3}$ is the state and $f(x) \in R^{3}$ is a continuous vector function.

According to the algebraic equation (23), it results in

$$
\left\{\begin{array}{l}
\alpha_{2} \alpha_{3}=\alpha_{1} \\
\alpha_{1} \alpha_{3}=\alpha_{2} \\
\alpha_{1} \alpha_{3}=\alpha_{2}
\end{array}\right.
$$

There are three solutions for equation (68), i.e.,

$$
\begin{aligned}
& \beta_{1}^{(2)}=\left(\begin{array}{l}
\alpha_{i_{1}} \\
\alpha_{i_{2}} \\
\alpha_{i_{3}}
\end{array}\right)=\left(\begin{array}{l}
\alpha_{2} \\
\alpha_{3} \\
\alpha_{1}
\end{array}\right)=\left(\begin{array}{c}
-1 \\
-1 \\
1
\end{array}\right), \\
& \beta_{2}^{(2)}=\left(\begin{array}{l}
\alpha_{i_{1}} \\
\alpha_{i_{3}} \\
\alpha_{i_{2}}
\end{array}\right)=\left(\begin{array}{l}
\alpha_{1} \\
\alpha_{3} \\
\alpha_{2}
\end{array}\right)=\left(\begin{array}{c}
-1 \\
-1 \\
1
\end{array}\right), \\
& \beta_{3}^{(2)}=\left(\begin{array}{l}
\alpha_{i_{1}} \\
\alpha_{i_{2}} \\
\alpha_{i_{3}}
\end{array}\right)=\left(\begin{array}{l}
\alpha_{1} \\
\alpha_{2} \\
\alpha_{3}
\end{array}\right)=\left(\begin{array}{c}
-1 \\
-1 \\
1
\end{array}\right) .
\end{aligned}
$$

For the solution given in equation (69), $\alpha_{1}=1$ and $\alpha_{2}=\alpha_{3}=-1$.

By Algorithm 1, it results in

$$
T=\left(\begin{array}{c}
\delta_{3}^{i_{1}} \\
\delta_{3}^{i_{2}} \\
\delta_{3}^{i_{3}}
\end{array}\right)=\left(\begin{array}{l}
\delta_{3}^{2} \\
\delta_{3}^{3} \\
\delta_{3}^{1}
\end{array}\right)=\left(\begin{array}{lll}
0 & 1 & 0 \\
0 & 0 & 1 \\
1 & 0 & 0
\end{array}\right) .
$$

Thus, let $X^{E}=\left(X_{1}^{E}, X_{2}^{E}\right)^{T}=\left(x_{2}, x_{3}\right)^{T}, X^{e}=x_{1}$; then, the Chen-Lee system (67) is rewritten as follows: 


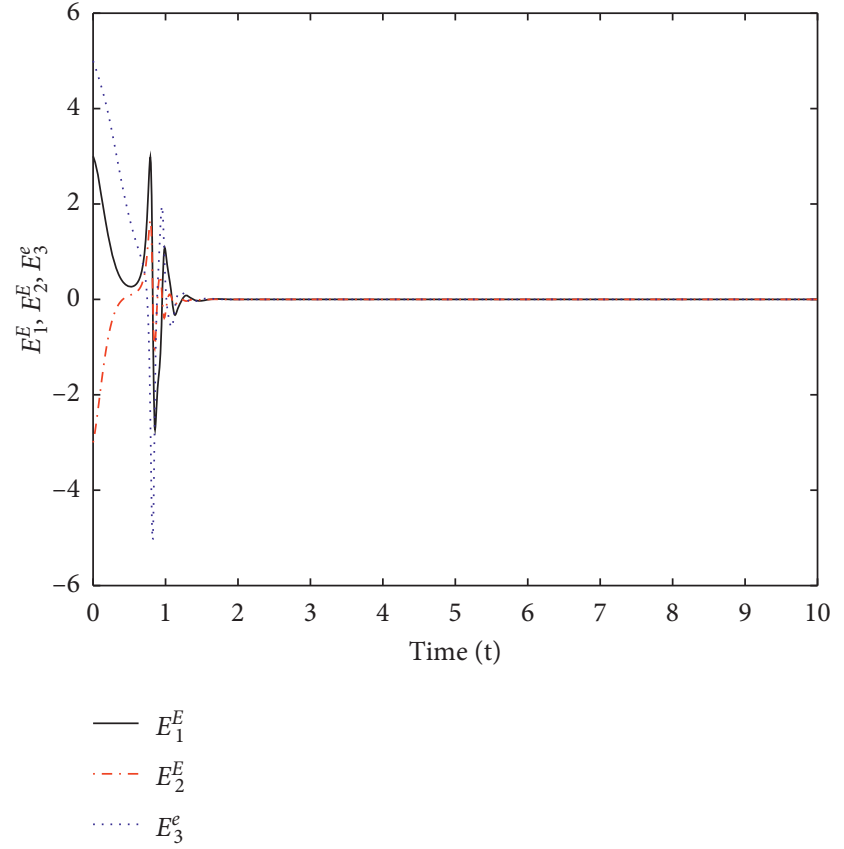

FIgURE 4: The sum and error system is asymptotically stable.

$$
\begin{aligned}
\dot{X}^{E} & =F^{E}\left(X^{E}, X^{e}\right), \\
\dot{X}^{e} & =F^{e}\left(X^{E}, X^{e}\right), \\
F^{E}\left(X^{E}, X^{e}\right) & =M\left(X^{e}\right) X^{E}, \\
M\left(X^{e}\right) & =\left(\begin{array}{cc}
-10 & X^{e} \\
\frac{1}{3} X^{e} & -3.8
\end{array}\right), \\
F^{e}\left(X^{E}, X^{e}\right) & =5 X^{e}-X_{1}^{E} X_{2}^{E},
\end{aligned}
$$

and $F^{e}\left(X^{E}, X^{e}\right)=F^{e}\left(-X^{E}, X^{e}\right)$.

Next, the slave Chen-Lee system is given as

$$
\begin{aligned}
\dot{Y}^{E} & =F^{E}\left(Y^{E}, Y^{e}\right)+B^{E} u, \\
\dot{Y}^{e} & =F^{e}\left(Y^{E}, Y^{e}\right)+B^{e} u, \\
F^{E}\left(Y^{E}, Y^{e}\right) & =M\left(Y^{e}\right) Y^{E}, \\
M\left(Y^{e}\right) & =\left(\begin{array}{cc}
-10 & Y^{e} \\
\frac{1}{3} Y^{e} & -3.8
\end{array}\right), \\
F^{e}\left(Y^{E}, Y^{e}\right) & =5 Y^{e}-Y_{1}^{E} Y_{2}^{E}, \\
B^{E} & =\left(\begin{array}{ll}
0 & 0 \\
1 & 0
\end{array}\right), \\
B^{e} & =\left(\begin{array}{ll}
0 & 1
\end{array}\right),
\end{aligned}
$$

and $F^{e}\left(Y^{E}, Y^{e}\right)=F^{e}\left(-Y^{E}, Y^{e}\right)$; the controller $u$ is designed as follows:
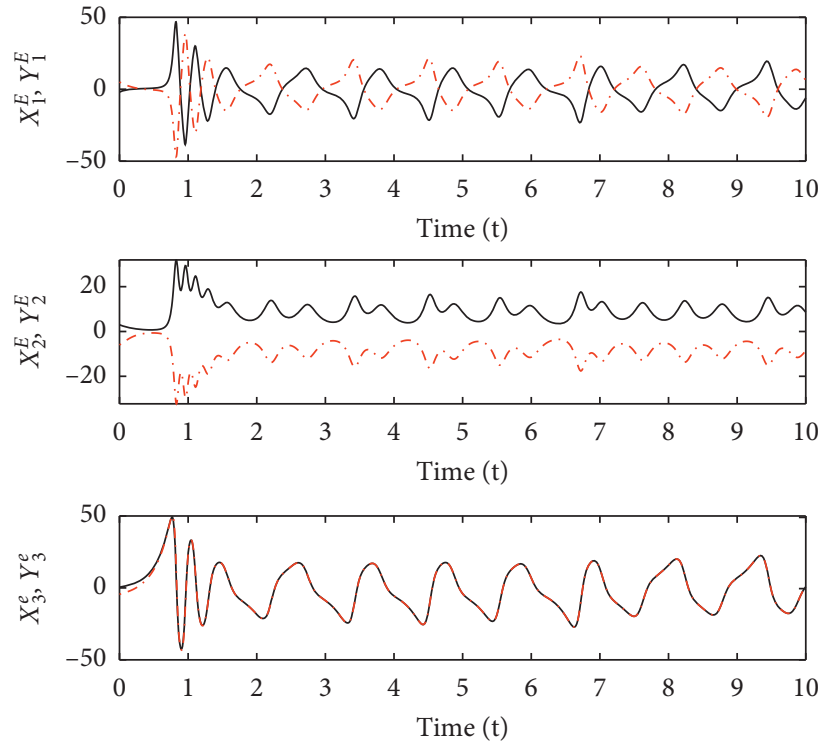

Figure 5: $X_{1}^{E}, X_{2}^{E}, X_{3}^{e}$ and $Y_{1}^{E}, Y_{2}^{E}, Y_{3}^{e}$, respectively.

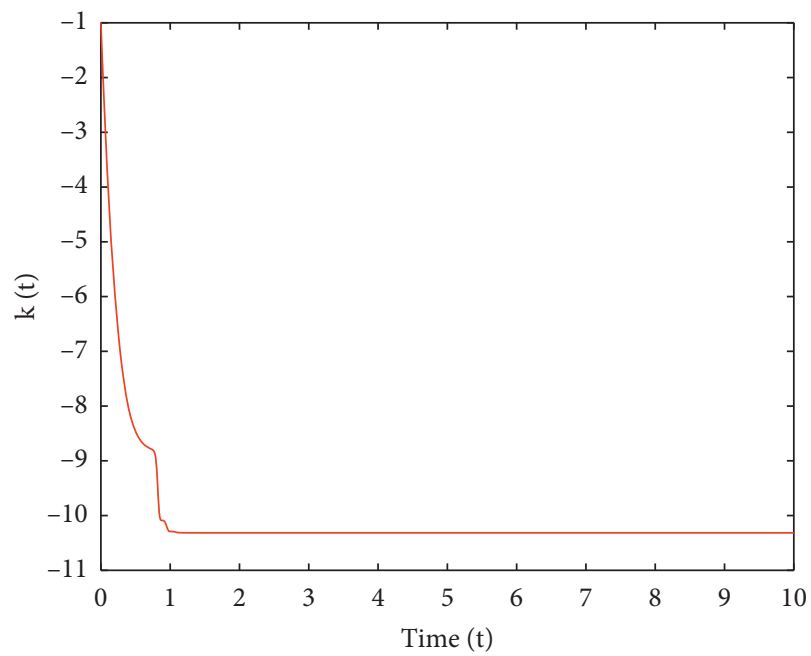

FIgURE 6: $k(t)$ tends to a constant.

$$
u=K E=k(t) B^{T} E=k(t)\left(\begin{array}{lll}
0 & 1 & 0 \\
0 & 0 & 1
\end{array}\right) E=\left(\begin{array}{c}
k(t) E_{2}^{E} \\
k(t) E_{3}^{e}
\end{array}\right)
$$

and $\dot{k}(t)=-\|E\|^{2}$.

Similarly, for this example, numerical simulation is conducted by setting following initial conditions: $x_{1}(0)=1.2, x_{2}(0)=-3.1, \quad x_{3}(0)=1.2$, $y_{1}(0)=4.8, y_{2}(0)=-3.2, y_{3}(0)=7.6, \quad$ and $\quad k(0)=-1$. Figure 4 shows that the sum and error system is asymptotically stable; Figure 5 shows the states $X_{1}^{E}, X_{2}^{E}, X_{3}^{e}$ and $Y_{1}^{E}, Y_{2}^{E}, Y_{3}^{e}$, respectively. Figure 6 shows that the feedback gain $k(t)$ tends to a constant.

For solutions given in equations (70) and (71), similar results can be obtained by the same procedure: 


\section{Conclusions}

In this paper, we investigated a new synchronization phenomenon that is the simultaneity of synchronization and antisynchronization for a class of chaotic systems. We developed a systematic method that can be used for proving the existence of the simultaneity of synchronization and antisynchronization, deriving all solutions for this type of synchronization phenomenon and then designing the corresponding physical controllers. To verify the validity and effectiveness of the theoretical results, two illustrative examples based on numerical simulations were provided.

In the future, microcontroller-based applications and predefined-time chaos synchronization problem need to be developed based on the results of this study.

\section{Data Availability}

No data were used to support this study. In our paper, we only use MATLAB for simulation. Therefore, we can only provide simulation programming, which can be obtained from the corresponding author upon request.

\section{Conflicts of Interest}

The authors declare that there are no conflicts of interest regarding the publication of this paper.

\section{Acknowledgments}

This work is supported by the Shandong Province Key Research and Development Program (Grant No. 2019GGX101056), the Natural Science Foundation of Shandong Province (Grant No. ZR2018MF016).

\section{References}

[1] L. M. Pecora and T. L. Carroll, "Synchronization in chaotic systems," Physical Review Letters, vol. 64, no. 8, pp. 821-824, 1990.

[2] E. Ott, C. Grebogi, and J. A. Yorke, "Controlling chaos," Physical Review Letters, vol. 64, no. 11, pp. 1196-1199, 1990.

[3] R. Guo, "A simple adaptive controller for chaos and hyperchaos synchronization," Physics Letters A, vol. 372, no. 34, pp. 5593-5597, 2008.

[4] A. Göksu, U. E. Kocamaz, and Y. Uyaroğlu, "Synchronization and control of chaos in supply chain management," Computers \& Industrial Engineering, vol. 86, pp. 107-115, 2015.

[5] X. Yi, R. Guo, and Y. Qi, "Stabilization of chaotic systems with both uncertainty and disturbance by the UDE-based control method," IEEE Access, vol. 8, no. 1, pp. 62471-62477, 2020.

[6] G. Wang, "Stabilization and synchronization of genesio-tesi system via single variable feedback controller," Physics Letters A, vol. 374, no. 28, pp. 2831-2834, 2010.

[7] J. Sieber, E. O. Chenko, and M. Wolfrum, "Controlling unstable chaos: stabilizing chimera states by feedback," Physical Review Letter, vol. 112, no. 5, 2014.

[8] D. Meng and Y. Li, "Adaptive synchronization of 4-dimensional energy resource unknown time-varying delay systems," IEEE Access, vol. 5, pp. 21258-21263, 2017.

[9] T. Wang, D. Wang, and K. Wu, "Chaotic adaptive synchronization control and application in chaotic secure communication for industrial internet of things," IEEE Access, vol. 6, pp. 8584-8590, 2018.

[10] Z. Wang, Y. Sun, and B. Liang, "Synchronization control for bilateral teleoperation system with position error constraints: a fixed-time approach," ISA Transactions, vol. 93, pp. 125-136, 2019.

[11] L. Liu, R. Guo, J. Ji, Z. H. Miao, and J. Zhou, "Practical consensus tracking control of multiple nonholonomic wheeled mobile robots in polar coordinates," International Journal of Robust Nonlinear Control, vol. 30, no. 10, pp. 3831-3847, 2020.

[12] W. Wang, X. Jia, Z. Wang et al., "Fixed-time synchronization of fractional order memristive mam neural networks by sliding mode control," Neurocomputing, vol. 401, no. 8, pp. 364-376, 2020.

[13] M. Luo and M. Pourgholi, "Robust fixed-time synchronization of fractional order chaotic using free chattering nonsingular adaptive fractional sliding mode controller design," Chaos, Solitons \& Fractals, vol. 113, no. 8, pp. 135-147, 2018.

[14] A. M. Alimi, C. Aouiti, and E. A. Assali, "Finite-time and fixed-time synchronization of a class of inertial neural networks with multi-proportional delays and its application to secure communication," Neurocomputing, vol. 332, no. 3, pp. 29-43, 2019.

[15] H. Jiang, Y. Liu, L. Zhang, and J. Yu, "Anti-phase synchronization and symmetry-breaking bifurcation of impulsively coupled oscillators," Communications in Nonlinear Science and Numerical Simulation, vol. 39, no. 10, pp. 199-208, 2016.

[16] L. Ren and R. Guo, "Synchronization and antisynchronization for a class of chaotic systems by a simple adaptive controller," Mathematical Problems in Engineering, vol. 2015, Article ID 434651, 7 pages, 2015.

[17] R. Guo, "Projective synchronization of a class of chaotic systems by dynamic feedback control method," Nonlinear Dynamics, vol. 90, no. 1, pp. 53-64, 2017.

[18] G.-D. Lin, Y.-T. Gao, L. Wang, D. X. Meng, and X. Yu, "Elastic-inelastic-interaction coexistence and double wronskian solutions for the whitham-broer-kaup shallow-waterwave model," Communications in Nonlinear Science and Numerical Simulation, vol. 16, no. 8, pp. 3090-3096, 2011.

[19] I. Aviram and A. Rabinovitch, "Bifurcation analysis of bacteria and bacteriophage coexistence in the presence of bacterial debris," Communications in Nonlinear Science and Numerical Simulation, vol. 17, no. 1, pp. 242-254, 2012.

[20] W. Jiang and B. Niu, "On the coexistence of periodic or quasiperiodic oscillations near a hopf-pitchfork bifurcation in NFDE," Communications in Nonlinear Science and Numerical Simulation, vol. 18, no. 3, pp. 464-477, 2013.

[21] J. Kengne, Z. Njitacke Tabekoueng, and H. B. Fotsin, "Coexistence of multiple attractors and crisis route to chaos in autonomous third order duffing-holmes type chaotic oscillators," Communications in Nonlinear Science and Numerical Simulation, vol. 36, no. 7, pp. 29-44, 2016.

[22] A. Naimzada and M. Pireddu, "An evolutive discrete exchange economy model with heterogeneous preferences," Chaos, Solitons \& Fractals, vol. 111, no. 12, pp. 35-43, 2018.

[23] Q. Zhang, J. Lü, and S. Chen, "Coexistence of anti-phase and complete synchronization in the generalized lorenz system," Communications in Nonlinear Science and Numerical Simulation, vol. 15, no. 10, pp. 3067-3072, 2010.

[24] E. N. Lorenz, "Deterministic nonperiodic flow," Journal of Atmospheric Science, vol. 20, no. 2, pp. 130-141, 1963.

[25] J.-H. Chen, "Controlling chaos and chaotification in the chenlee system by multiple time delays," Chaos, Solitons \& Fractals, vol. 36, no. 4, pp. 843-852, 2008. 